\title{
Studies of X-ray burst reactions with radioactive ion beams from RESOLUT
}

\author{
J. C. Blackmon ${ }^{1, \star}$, I. Wiedenhöver ${ }^{2}$, J. Belarge ${ }^{2}$, S. A. Kuvin ${ }^{2}$, M. Anastasiou ${ }^{2}$, L. T. Baby ${ }^{2}$, J. \\ Baker $^{2}$, K. Colbert ${ }^{3}$, C. M. Deibel ${ }^{1}$, H. E. Gardiner ${ }^{1}$, D. L. Gay ${ }^{3}$, E. Good ${ }^{1}$, P. Höflich², A. A. D. \\ Hood $^{1}$, N. Keely ${ }^{4}$, J. Lai ${ }^{1}$, A. Laminack ${ }^{1}$, L. E. Linhardt ${ }^{1}$, J. Lighthall ${ }^{1,2}$, K. T. Macon ${ }^{1}$, E. Need ${ }^{1}$, \\ N. Quails ${ }^{3}$, B. C. Rasco ${ }^{1}, N$. Rijal ${ }^{2}$, and A. Volya ${ }^{2}$ \\ ${ }^{1}$ Louisiana State University, Baton Rouge, LA 70803 USA \\ ${ }^{2}$ Florida State University, Tallahassee, FL 32306 USA \\ ${ }^{3}$ University of North Florida, Jacksonville, FL 32224 USA \\ ${ }^{4}$ National Center for Nuclear Research, Otwock, Poland
}

\begin{abstract}
Reactions on certain proton-rich, radioactive nuclei have been shown to have a significant influence on X-ray bursts. We provide an overview of two recent measurements of important $\mathrm{X}$-ray burst reactions using in-flight radioactive ion beams from the RESOLUT facility at the J. D. Fox Superconducting Accelerator Laboratory at Florida State University. The ${ }^{17} \mathrm{~F}(\mathrm{~d}, \mathrm{n}){ }^{18} \mathrm{Ne}$ reaction was measured, and Asymptotic Normalization Coefficients were extracted for bound states in ${ }^{18} \mathrm{Ne}$ that determine the direct-capture cross section dominating the ${ }^{17} \mathrm{~F}(\mathrm{p}, \gamma){ }^{18} \mathrm{Ne}$ reaction rate for $T \lesssim 0.45 \mathrm{GK}$. Unbound resonant states were also studied, and the single-particle strength for the $4.523-\mathrm{MeV}\left(3^{+}\right)$ state was found to be consistent with previous results. The ${ }^{19} \mathrm{Ne}(\mathrm{d}, \mathrm{n}){ }^{20} \mathrm{Na}$ proton transfer reaction was used to study resonances in the ${ }^{19} \mathrm{Ne}(\mathrm{p}, \gamma)^{20} \mathrm{Na}$ reaction. The most important $2.65-\mathrm{MeV}$ state in ${ }^{20} \mathrm{Na}$ was observed to decay by proton emission to both the ground and first-excited states in ${ }^{19} \mathrm{Ne}$, providing strong evidence for a $3^{+}$spin assignment and indicating that proton capture on the thermally-populated first-excited state in ${ }^{19} \mathrm{Ne}$ is an important contributor to the ${ }^{19} \mathrm{Ne}(\mathrm{p}, \gamma)^{20} \mathrm{Na}$ reaction rate.
\end{abstract}

\section{Introduction}

$\mathrm{X}$-ray bursts occur in binary systems when hydrogen-rich matter from a main-sequence star accretes onto a neutron star and ignites under degenerate conditions. These are the most common stellar explosions, with over 100 systems known to exhibit bursts recurring with timescales typically from hours to days. Comparisons of observations of many bursts (e.g. see [1]) to improved computational simulations using a variety of astrophysical models (e.g. see [2]) are providing insights into the composition, dynamics, and evolution of these systems. Simulations also show that certain nuclear reactions involving proton-rich, radioactive nuclei have a direct impact on energy generation, nucleosynthesis, and the resulting light curve $[3,4]$. The rates of many of these reactions are purely theoretical or have large uncertainties due to experimental challenges in studying short-lived nuclei that hinder our understanding of X-ray bursts.

^e-mail: blackmon@1su.edu 
Some of the important reactions in X-ray bursts are those that involve breakout of the hot-CNO cycle, either through the ${ }^{17} \mathrm{~F}(\mathrm{p}, \gamma){ }^{18} \mathrm{Ne}(\alpha, \mathrm{p})^{21} \mathrm{Na}$ or ${ }^{15} \mathrm{O}(\alpha, \gamma){ }^{19} \mathrm{Ne}(\mathrm{p}, \gamma)^{20} \mathrm{Na}$ reaction sequences. We measured the ${ }^{17} \mathrm{~F}(\mathrm{~d}, \mathrm{n}){ }^{18} \mathrm{Ne}$ and ${ }^{19} \mathrm{Ne}(\mathrm{d}, \mathrm{n}){ }^{20} \mathrm{Na}$ reactions to improve our understanding of the ${ }^{17} \mathrm{~F}(\mathrm{p}, \gamma){ }^{18} \mathrm{Ne}$ and ${ }^{19} \mathrm{Ne}(\mathrm{p}, \gamma)^{20} \mathrm{Na}$ capture reactions, respectively, that are important in breakout from the hot-CNO cycle. Both measurements were conducted at the J. D. Fox Superconducting Accelerator Laboratory at Florida State University using "in-flight" radioactive ion beams. Stable beams of ${ }^{16} \mathrm{O}$ and ${ }^{19} \mathrm{~F}$ bombarded a cryogenic gas cell to produce ${ }^{17} \mathrm{~F}$ and ${ }^{19} \mathrm{Ne}$ from the ${ }^{16} \mathrm{O}(\mathrm{d}, \mathrm{n}){ }^{17} \mathrm{~F}$ and ${ }^{19} \mathrm{~F}(\mathrm{p}, \mathrm{n}){ }^{19} \mathrm{Ne}$ reactions, respectively. The radioactive products were collected and separated by the RESOLUT facility [5]. The secondary radioactive beams bombarded a deuterated polyethylene target, and a suite of detector systems was used to detect neutrons, gamma rays, light charged particles and heavy ions.

\section{$2{ }^{17} \mathbf{F}(\mathbf{p}, \gamma){ }^{18} \mathrm{Ne}$}

The ${ }^{17} \mathrm{~F}(\mathrm{p}, \gamma){ }^{18} \mathrm{Ne}$ reaction rate is determined mainly by contributions from direct capture and a single $3^{+}$resonance at $E_{c m}=600 \mathrm{keV}$. The $600-\mathrm{keV}$ resonance strength was directly measured at the Holifield Radioactive Ion Beam Facility [6], but the direct-capture contribution that dominates the reaction rate at $T \lesssim 0.45 \mathrm{GK}$ remains uncertain. A measurement of the ${ }^{17} \mathrm{~F}(\mathrm{p}, \gamma){ }^{18} \mathrm{Ne}$ direct-capture cross section at $E_{c m}<600 \mathrm{keV}$ would require a ${ }^{17} \mathrm{~F}$ beam intensity of about $10^{9} \mathrm{~s}^{-1}$. Such intensities are beyond the reach of current facilities, and indirect techniques must be applied to better constrain the direct-capture cross section.

We measured the ${ }^{17} \mathrm{~F}(\mathrm{~d}, \mathrm{n}){ }^{18} \mathrm{Ne}$ proton-transfer reaction to study states in ${ }^{18} \mathrm{Ne}$ that are important for the ${ }^{17} \mathrm{~F}(\mathrm{p}, \gamma){ }^{18} \mathrm{Ne}$ reaction rate, including bound states that determine the direct-capture cross section [7]. A 95.5-MeV beam of ${ }^{17} \mathrm{~F}$ from RESOLUT with an intensity of about $3 \times 10^{4} \mathrm{~s}^{-1}$ bombarded a $0.52 \mathrm{mg} / \mathrm{cm}^{2} \mathrm{CD}_{2}$ target. Neutrons from the $(\mathrm{d}, \mathrm{n})$ reaction were detected at $\theta_{\text {lab }}=145^{\circ}-165^{\circ}$ using the RESONEUT array of P-terphenyl scintillators that provided good $n-\gamma$ discrimination for energies greater than about $50 \mathrm{keV}_{\text {ee }}$ [8]. All heavy ions (beam particles and heavy reaction products) were detected using a fast-counting, position-sensitive gas ionization detector covering $\theta_{\text {lab }}<6^{\circ}$ that determined the atomic number through relative energy loss and measured the position (in 2 dimensions perpendicular to the beam axis) with a resolution of better than $3 \mathrm{~mm}$ [9]. Gamma rays emitted from bound, excited states were detected with a total efficiency of $12 \%$ using an array of $20 \mathrm{NaI}(\mathrm{Tl})$ scintillators [10] arranged in a barrel configuration subtending $\theta_{\mathrm{lab}}=35^{\circ}-100^{\circ}$. The triple coincidence of neutrons, $\gamma$ rays, and heavy ions identified by atomic number provided clean selection of the population of bound states in ${ }^{18} \mathrm{Ne}$ except for the ground state, which is expected to be weakly populated.

The relative timing between the neutrons and the accelerator $r f$ signal was used to determine the $E_{x}$ of states in ${ }^{18} \mathrm{Ne}$ by time-of-flight with a corresponding energy resolution of about $\Delta E_{c m} \approx$ $150 \mathrm{keV}$. For a triple coincidence of $n-\gamma-{ }^{18} \mathrm{Ne}$, we observe two peaks in the time-of-flight spectrum corresponding to the $1.887-\mathrm{MeV}\left(2^{+}\right)$state and to the $4^{+}-0^{+}-2^{+}$triplet of states at about $3.5 \mathrm{MeV}$. While the triplet is not resolved, population of the $3.57-\mathrm{MeV}\left(0^{+}\right)$state is expected to be very weak. Assuming the $0^{+}$to be negligible, we determined the cross sections for population of the $1.887-\mathrm{MeV}$ $\left(2^{+}\right), 3.376-\mathrm{MeV}\left(4^{+}\right)$and $3.616-\mathrm{MeV}\left(2^{+}\right)$states from a combined fit to the neutron time-of-flight spectrum (see Fig. 13 in [7]). These cross sections (corresponding to very forward angles for $\theta_{c m}$ ) determine Asymptotic Normalization Coefficients for the states.

Direct capture is expected to be dominated by $\ell=0$ capture to the $2^{+}$states in ${ }^{18} \mathrm{Ne}$ below the proton threshold. We are unable to constrain the relative population of $s_{1 / 2}$ and $d_{5 / 2}$ for the $2^{+}$states from our data due to the limited statistics and angular range of the detected neutrons. Assuming the same relative $s_{1 / 2}$ and $d_{5 / 2}$ mixing as in the mirror states in ${ }^{18} \mathrm{O}$ [11], we find $C_{s_{1 / 2}}^{2}=16 \pm 8 \mathrm{fm}^{-1}$ for the 
1.887-MeV state and $C_{s_{1 / 2}}^{2}=150 \pm 60 \mathrm{fm}^{-1}$ for the 3.616-MeV state, assuming the reaction to occur at an average energy of $5.53 \mathrm{MeV} / \mathrm{u}$ corresponding to the center of the target. Using these values, we calculated the contribution to the ${ }^{17} \mathrm{~F}(\mathrm{p}, \gamma){ }^{18} \mathrm{Ne}$ reaction rate from direct capture. We find a $30 \%$ larger contribution from direct capture than estimated from properties of the mirror [12], though the results are consistent within uncertainties, which are dominated in our result by a statistical uncertainty of $30 \%$ in the cross section for populating the $3.616-\mathrm{MeV}$ state.

We were also able to study resonant states above the proton-separation energy, even though they predominantly decay by proton emission, providing no $\gamma$-ray signature and giving the same species of recoiling heavy ion as the beam $\left({ }^{17} \mathrm{~F}\right)$. We cleanly selected the events of interest in this case by identifying the emitted protons in an annular telescope array of silicon-strip detectors subtending $\theta_{\text {lab }}=8^{\circ}-21^{\circ}$. Correlating the measured protons with the ${ }^{17} \mathrm{~F}$ ions in the gas ionization chamber allowed proton-unbound states in ${ }^{18} \mathrm{Ne}$ to be accurately reconstructed from an invariant mass analysis using only the position and energy of the protons and ${ }^{17} \mathrm{~F}$ ions, thus inferring information about the outgoing neutron without using data from the RESONEUT neutron detector array. We do find consistent results when requiring coincidence neutron detection with RESONEUT, though the efficiency reduces the statistics by a factor of about 30 . The reconstructed excitation spectrum in ${ }^{18} \mathrm{Ne}$ from ${ }^{17} \mathrm{~F}+p$ coincidences (see Fig. 8 in [7]) is dominated by one strong resonance corresponding to the $3^{+}$state in ${ }^{18} \mathrm{Ne}$ at $4.523 \mathrm{MeV}$ that known to be the strongest resonance in the ${ }^{17} \mathrm{~F}(\mathrm{p}, \gamma){ }^{18} \mathrm{Ne}$ reaction [6]. The higher statistics achieved without neutron detection allowed the distribution of events in angle/energy to be compared to a FRESCO coupled-channels calculation. We find dominant population of the state through $\ell=0$ transfer, confirming the $3^{+}$spin assignment, with a single-particle spectroscopic factor of $C^{2} S=0.78 \pm 0.06$ corresponding to a proton partial width of $\Gamma_{p}=(14.2 \pm 1.1) \mathrm{keV}$, where the uncertainty is dominated by the systematic uncertainties in the analysis. The extracted partial width is about $20 \%$ lower than determined from ${ }^{17} \mathrm{~F}+p$ elastic scattering [13]. It should be noted that the triple coincidence of neutrons, protons, and ${ }^{17} \mathrm{~F}$ ions showed strong population of this resonant state in the neutron time-of-flight spectrum, serving as a check on the time-of-flight analysis for the bound states.

\section{$3{ }^{19} \mathrm{Ne}(\mathbf{p}, \gamma){ }^{20} \mathrm{Na}$}

The ${ }^{19} \mathrm{Ne}(\mathrm{p}, \gamma)^{20} \mathrm{Na}$ reaction rate is dominated by contributions from resonances corresponding to states just above the proton-separation energy $\left(S_{p}=2.19 \mathrm{MeV}\right)$ in ${ }^{20} \mathrm{Na}$. While the structure of ${ }^{20} \mathrm{Na}$ is relatively well established for bound states, properties of proton-unbound levels are uncertain. The level scheme is shown in Figure 1 compared to the isospin-mirror nucleus ${ }^{20} \mathrm{~F}$, where we have included spin-parity $\left(J^{\pi}\right)$ information only when unambiguous. There are 6 observed states in ${ }^{20} \mathrm{Na}$ within $1 \mathrm{MeV}$ above the proton-separation energy. Excitation energies are relatively well determined from charge-exchange reactions, but these studies have placed only weak constraints on $J^{\pi}$ values due to challenges from unresolved states and increased background above the separation energy, problems exacerbated by difficulties with neon targets [14-16]. Only the $3.00-\mathrm{MeV}\left(1^{+}\right)$and $3.09-\mathrm{MeV}$ $\left(0^{+}\right)$states have clear assignments based upon ${ }^{19} \mathrm{Ne}+p$ elastic scattering [17] and their population in allowed $\beta$-decay transitions from ${ }^{20} \mathrm{Mg}[18,19]$. These states have been identified as mirror levels to the $3.49-\mathrm{MeV}$ and $3.53-\mathrm{MeV}$ states in ${ }^{20} \mathrm{~F}$, with a large Coulomb shift resulting from a substantial $2 s_{1 / 2}$ component in the wavefunction as expected from $s d$ shell model calculations.

The other levels at $2.65,2.85,2.98$ and $3.07 \mathrm{MeV}$ in ${ }^{20} \mathrm{Na}$ have generally been assumed to be mirrors to the only 4 states observed in ${ }^{20} \mathrm{~F}$ in a comparable energy range [20]. Only the 2.966-MeV state in ${ }^{20} \mathrm{~F}$ has majority $s d$ single-particle character, being strongly populated in the ${ }^{19} \mathrm{~F}(\mathrm{~d}, \mathrm{p})^{20} \mathrm{~F}$ and ${ }^{18} \mathrm{O}\left({ }^{3} \mathrm{He}, \mathrm{p}\right)^{20} \mathrm{~F}$ reactions with angular distributions giving a $3^{+}$assignment [21]. The other 3 states in ${ }^{20} \mathrm{~F}$ have more complex configurations. The $2.864-\mathrm{MeV}$ and $3.171-\mathrm{MeV}$ states in ${ }^{20} \mathrm{~F}$ are weakly 
populated in transfer reactions. Angular distributions indicate negative parity for the $2.864-\mathrm{MeV}$ state, with arguments favoring a $3^{-}$assignment [22]. The $3.171-\mathrm{MeV}$ state is populated predominantly by $\ell=2$ transfer in both ${ }^{19} \mathrm{~F}(\mathrm{~d}, \mathrm{p}){ }^{20} \mathrm{~F}$ and ${ }^{18} \mathrm{O}\left({ }^{3} \mathrm{He}, \mathrm{p}\right){ }^{20} \mathrm{~F}$, indicating positive-parity and a likely $1^{+}$ assignment, but composed primarily of $6 p-2 h$ core excitations. Finally, the $2.968-\mathrm{MeV}$ state is a high-spin state, likely $J^{\pi} \geq 4^{-}$[23].

Most important is to determine the properties of the first state above the separation energy in ${ }^{20} \mathrm{Na}$ at $2.65 \mathrm{MeV}$ that could dominate the ${ }^{19} \mathrm{Ne}(\mathrm{p}, \gamma)^{20} \mathrm{Na}$ reaction rate given the low $\left(E_{c m}=457 \mathrm{keV}\right)$ resonance energy. Some arguments favor a $1^{+}$assignment for this state but with a small singleparticle component (see [24] for example). This is also supported an upper limit on the protoncapture resonance strength $(\omega \gamma<16 \mathrm{meV})$ set from the a ${ }^{19} \mathrm{Ne}(\mathrm{p}, \gamma)^{20} \mathrm{Na}$ reaction measurement [25]. However, some information argues otherwise. The lack of observation of the $2.65-\mathrm{MeV}$ state in ${ }^{20} \mathrm{Mg}$ beta-decay strongly favors a forbidden transition [19], and other charge-exchange measurements have argued for a $3^{+}$assignment $[26,27]$. These charge-exchange studies also indicated a tentative $3^{-}$ assignment of the $2.85-\mathrm{MeV}$ state in ${ }^{20} \mathrm{Na}$ as a potential mirror to the $2.86-\mathrm{MeV}$ level in ${ }^{20} \mathrm{~F}$, though these states are not well resolved from neighboring states and are weakly populated.

We studied the properties of unbound states in ${ }^{20} \mathrm{Na}$ using the ${ }^{19} \mathrm{Ne}(\mathrm{d}, \mathrm{n}){ }^{20} \mathrm{Na}$ reaction [28]. The experimental configuration and approach were similar to that in the study of unbound levels in ${ }^{18} \mathrm{Ne}$. A $86-\mathrm{MeV}$ beam of ${ }^{19} \mathrm{Ne}$ from RESOLUT bombarded a $0.52 \mathrm{mg} / \mathrm{cm}^{2} \mathrm{CD}_{2}$ target. Protons were detected and identified in an annular telescope of silicon strip detectors subtending $\theta_{\text {lab }}=8^{\circ}-21^{\circ}$ while heavy ions were detected using the position-sensitive gas ionization detector covering $\theta_{\mathrm{lab}}<6^{\circ}$. The ${ }^{19} \mathrm{Ne}$ beam intensity $\left(<2000 s^{-1}\right)$ was more than an order of magnitude less intense than in the ${ }^{17} \mathrm{~F}(\mathrm{~d}, \mathrm{n}){ }^{18} \mathrm{Ne}$ study described previously, which prohibited the coincident detection of neutrons due to the reduced efficiency. As with the ${ }^{18} \mathrm{Ne}$, however, we are able to reconstruct the unbound states of interest from an invariant mass analysis using the kinematics of the protons and ${ }^{19} \mathrm{Ne}$ ions (see Fig. 1 in [28]) with a center-of-mass energy resolution of about $200 \mathrm{keV}$ (FWHM)

One prominent feature in our reconstructed spectrum is a well-resolved resonance at $E_{c m}=$ $220 \mathrm{keV}$. There is no known level in ${ }^{20} \mathrm{Na}$ near $E_{x}=2.41 \mathrm{MeV}$ that would correspond to proton emission to the ground state of ${ }^{19} \mathrm{Ne}$ for this resonance. However, the $E_{c m}$ matches proton decay from the important $2.65-\mathrm{MeV}$ state in ${ }^{20} \mathrm{Na}$ to the first-excited state $\left(\frac{5}{2}^{+}\right)$in ${ }^{19} \mathrm{Ne}$. Emission of a $220-\mathrm{keV}$ proton strongly argues for $\ell=0$ angular momentum due to the prohibitive penetrability for larger $\ell$ values. An $\ell=2$ proton-decay branch at this energy would be about an order of magnitude weaker than the expected gamma-decay branch and would not be observed. This supports the $3^{+}$assignment for the $2.65-\mathrm{MeV}$ resonance as indicated from the weak population in beta decay. We also extract an angle/energy distribution for the emitted ${ }^{20} \mathrm{Na}$ (and inferred neutron) and model the ${ }^{19} \mathrm{Ne}(\mathrm{d}, \mathrm{n}){ }^{20} \mathrm{Na}$ cross section following the procedure of [29]. A fit to the angular distribution using $\ell=2$ protontransfer onto the ${ }^{19} \mathrm{Ne}$ ground state (required to populate a $3^{+}$resonant state) results in $\chi^{2} / v=1.8$, while a corresponding fit using $\ell=0$ proton transfer (likely for a $1^{+}$resonance) results in $\chi^{2} / v=4.3$. Both the angular distribution and the strength by which the first-excited state is populated in proton emission provides strong evidence supporting the $3^{+}$assignment for the $2.65-\mathrm{MeV}$ level.

The second prominent feature in our $E_{c m}$ spectrum is a strong resonance at $660 \mathrm{keV}$, corresponding to proton emission from the $2.85-\mathrm{MeV}$ state in ${ }^{20} \mathrm{Na}$ to the ground state in ${ }^{19} \mathrm{Ne}$. This resonance has a significant low-energy shoulder best described by two additional weaker resonances at $E_{c m}=440 \mathrm{keV}$ and $540 \mathrm{keV}$. The $440-\mathrm{keV}$ resonance corresponds to proton emission from the $2.65-\mathrm{MeV}$ state in ${ }^{20} \mathrm{Na}$ to the ${ }^{19} \mathrm{Ne}$ ground state. We find the branching ratio for proton decay from the $2.65-\mathrm{MeV}$ state to the ${ }^{19} \mathrm{Ne}$ ground state to be approximately equal to that for decay to the first-excited state. These equal decay branches imply that the wavefunction for the $2.65-\mathrm{MeV}$ state has a $s_{1 / 2}$ proton component (coupled to the ${ }^{19} \mathrm{Ne}$ first-excited state) with $C^{2} S=0.5$, about 7 times greater than the $d_{5 / 2}$ component, which is consistent with the $3^{+}$mirror state in ${ }^{20} \mathrm{~F}$ at $2.966 \mathrm{MeV}$. 


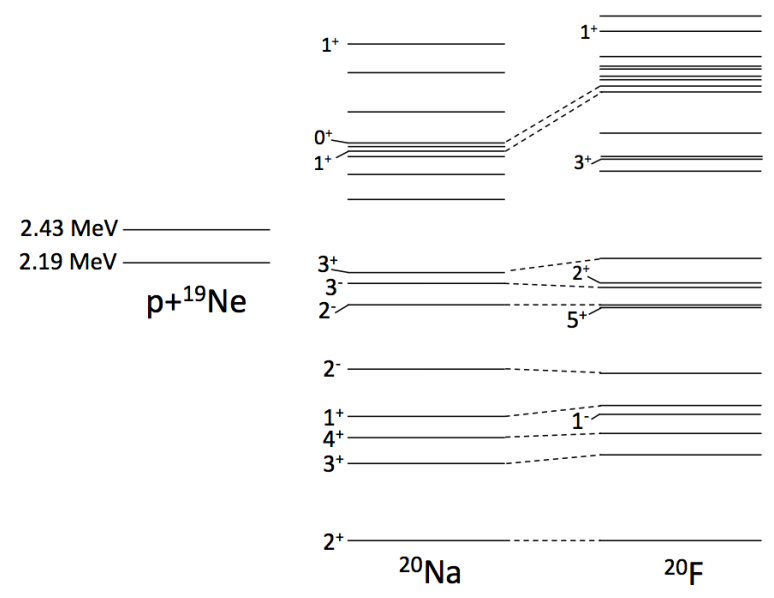

Figure 1. Level scheme of ${ }^{20} \mathrm{Na}$ compared to the mirror nucleus ${ }^{20} \mathrm{~F}$. Figure is adapted from the evaluation of [31]. The ${ }^{19} \mathrm{Ne}$ ground state $\left(J^{\pi}=1 / 2^{+}\right)$couples to an $\ell=0$ proton with channel spin $S=0,1$ corresponding to $E_{x}=2.19 \mathrm{MeV}$ in ${ }^{20} \mathrm{Na}$, while the first-excited state in ${ }^{19} \mathrm{Ne}\left(J^{\pi}=5 / 2^{+}\right)$couples to an $\ell=0$ proton with channel spin $S=2,3$ corresponding to $E_{x}=2.43 \mathrm{MeV}$ in ${ }^{20} \mathrm{Na}$.

The strong coupling of the $2.65-\mathrm{MeV}$ state to the first-exited state in ${ }^{19} \mathrm{Ne}$ significantly increases the ${ }^{19} \mathrm{Ne}(\mathrm{p}, \gamma){ }^{20} \mathrm{Na}$ reaction rate. The first-excited state in ${ }^{19} \mathrm{Ne}$ is sufficiently low in excitation energy that significant thermal population is possible (for example, a few percent at $T=1 G K$ ). The lower energy required for proton capture onto the thermally-excited ${ }^{19} \mathrm{Ne} \frac{5}{2}^{+}$state results in a contribution to the reaction rate that is comparable to that of capture on the ground state. Including this effect, we find a ${ }^{19} \mathrm{Ne}(\mathrm{p}, \gamma)^{20} \mathrm{Na}$ reaction rate that is significantly greater than previously estimated.

The strong population of the $2.85-\mathrm{MeV}$ state in our measurement is somewhat harder to interpret. Analyzing angular distributions for the ${ }^{19} \mathrm{Ne}(\mathrm{d}, \mathrm{n})^{20} \mathrm{Na}$ reaction results in $\chi^{2} / v=3.5$ for $\ell=0$ proton transfer and $\chi^{2} / v=5.1$ for $\ell=2$ proton transfer, with the $\ell=0$ fit giving a $s_{1 / 2}$ proton spectroscopic factor that is consistent only with the the $3.49-\mathrm{MeV}\left(1^{+}\right)$mirror state in ${ }^{20} \mathrm{~F}\left(C^{2} S=0.40\right)$ [30]. However, population of the $2.85-\mathrm{MeV}$ state is not observed in ${ }^{20} \mathrm{Mg}$ beta decay, and the decay branch must be on the order of $0.1 \%$ [19]. This is very unlikely for an allowed Gamow-Teller transition, and difficult to reconcile with the strong population of the $2.85-\mathrm{MeV}$ state that we observe in ${ }^{19} \mathrm{Ne}(\mathrm{d}, \mathrm{n}){ }^{20} \mathrm{Na}$. Further measurements are required to resolve the nature of the $2.85-\mathrm{MeV}$ state, but it is important to note that even with a $1^{+}$assignment, the $2.85-\mathrm{MeV}$ state contributes no more than a few percent to the overall ${ }^{19} \mathrm{Ne}(\mathrm{p}, \gamma){ }^{20} \mathrm{Na}$ reaction rate.

This work was partially supported by the National Science Foundation, under Grants No. PHY1401574, No. PHY-1064819, No. PHY-1126345 and partially supported by the U.S. Department of Energy, Office of Science, Office of Nuclear Physics under Grants No. DE-FG02-02ER41220 and No. DE-FG02-96ER40978.

\section{References}

[1] D.K. Galloway, M.P. Muno, J.M. Hartman, D. Psaltis, D. Chakrabarty, The Astrophysical Journal Supplement Series 179, 360 (2008)

[2] N. Lampe, A. Heger, D.K. Galloway, The Astrophysical Journal 819, 46 (2016)

[3] A. Parikh, J. José, F. Moreno, C. Iliadis, The Astrophysical Journal Supplement Series 178, 110 (2008)

[4] R.H. Cyburt, A.M. Amthor, A. Heger, E. Johnson, L. Keek, Z. Meisel, H. Schatz, K. Smith, The Astrophysical Journal 830, 55 (2016) 
[5] I. Wiedenhöver et al., Studies of Exotic Nuclei at the Resolut Facility of Florida State University, in Proceedings, 5th International Conference on Fission and Properties of Neutron Rich Nuclei (ICFN5): Sanibel Island, Florida, USA, November 4-10, 2012 (2013), pp. 144-151

[6] K.A. Chipps, D.W. Bardayan, J.C. Blackmon, K.Y. Chae, U. Greife, R. Hatarik, R.L. Kozub, C. Matei, B.H. Moazen, C.D. Nesaraja et al., Phys. Rev. Lett. 102, 152502 (2009)

[7] S. Kuvin et al., submitted to Phys. Rev. C (under review)

[8] L. Baby et al., submitted to Nucl. Instrum. Methods Phys. Res. A (in press)

[9] J. Lai et al., submitted to Nucl. Instrum. Methods Phys. Res. A (under review)

[10] N. Kaloskamis, K. Chan, A. Chishti, J. Greenberg, C. Lister, S. Freedman, M. Wolanski, J. Last, B. Utts, Nuclear Instruments and Methods in Physics Research Section A: Accelerators, Spectrometers, Detectors and Associated Equipment 330, 447 (1993)

[11] T.K. Li, D. Dehnhard, R.E. Brown, P.J. Ellis, Phys. Rev. C 13, 55 (1976)

[12] T. Al-Abdullah, F. Carstoiu, X. Chen, H.L. Clark, C.A. Gagliardi, Y.W. Lui, A. Mukhamedzhanov, G. Tabacaru, Y. Tokimoto, L. Trache et al., Phys. Rev. C 89, 025809 (2014)

[13] D.W. Bardayan, J.C. Blackmon, C.R. Brune, A.E. Champagne, A.A. Chen, J.M. Cox, T. Davinson, V.Y. Hansper, M.A. Hofstee, B.A. Johnson et al., Phys. Rev. Lett. 83, 45 (1999)

[14] S. Kubono, H. Orihara, S. Kato, T. Kajino, Astrophys. J. 344, 460 (1989)

[15] N.M. Clarke, P.R. Hayes, M.B. Becha, C.N. Pinder, S. Roman, Journal of Physics G: Nuclear and Particle Physics 16, 1547 (1990)

[16] J. Görres, M. Wiescher, Phys. Rev. C 52, 412 (1995)

[17] R. Coszach, T. Delbar, W. Galster, P. Leleux, I. Licot, E. Liénard, P. Lipnik, C. Michotte, A. Ninane, J. Vervier et al., Phys. Rev. C 50, 1695 (1994)

[18] L.J. Sun, X.X. Xu, D.Q. Fang, C.J. Lin, J.S. Wang, Z.H. Li, Y.T. Wang, J. Li, L. Yang, N.R. Ma et al., Phys. Rev. C 95, 014314 (2017)

[19] J. Wallace, P. Woods, G. Lotay, A. Alharbi, A. Banu, H. David, T. Davinson, M. McCleskey, B. Roeder, E. Simmons et al., Physics Letters B 712, 59 (2012)

[20] J.P. Wallace, P.J. Woods, Phys. Rev. C 86, 068801 (2012)

[21] D.J. Crozier, H.T. Fortune, Phys. Rev. C 10, 1697 (1974)

[22] H.T. Fortune, R.R. Betts, Phys. Rev. C 10, 1292 (1974)

[23] J.C. Legg, D.J. Crozier, G.G. Seaman, H.T. Fortune, Phys. Rev. C 18, 2202 (1978)

[24] M. Smith, P. Magnus, K. Hahn, A. Howard, P. Parker, A. Champagne, Z. Mao, Nuclear Physics A 536, 333 (1992)

[25] M. Couder, C. Angulo, E. Casarejos, P. Demaret, P. Leleux, F. Vanderbist, Phys. Rev. C 69, 022801 (2004)

[26] N.M. Clarke, S. Roman, C.N. Pinder, P.R. Hayes, Journal of Physics G: Nuclear and Particle Physics 19, 1411 (1993)

[27] B.D. Anderson, B. Wetmore, A.R. Baldwin, L.A.C. Garcia, D.M. Manley, R. Madey, J.W. Watson, W.M. Zhang, B.A. Brown, C.C. Foster et al., Phys. Rev. C 52, 2210 (1995)

[28] J. Belarge, S.A. Kuvin, L.T. Baby, J. Baker, I. Wiedenhöver, P. Höflich, A. Volya, J.C. Blackmon, C.M. Deibel, H.E. Gardiner et al., Phys. Rev. Lett. 117, 182701 (2016)

[29] N. Keeley, N. Alamanos, V. Lapoux, Phys. Rev. C 69, 064604 (2004)

[30] H.T. Fortune, G.C. Morrison, R.C. Bearse, J.L. Yntema, B.H. Wildenthal, Phys. Rev. C 6, 21 (1972)

[31] D. Tilley, C. Cheves, J. Kelley, S. Raman, H. Weller, Nuclear Physics A 636, 249 (1998) 